\title{
CRASH 3 trial: "verum finalem" for role of tranexamic acid in brain trauma?
}

\author{
Dr. Amit Thapa MBBS, MS, MCh, IFAANS \\ Professor, Department of Neurological Surgery \\ Kathmandu Medical College Teaching Hospital \\ (KMCTH) \\ Sinamangal, Kathmandu, Nepal \\ ORCID iD: https://orcid.org/0000-0003-1896-3115
}

\author{
Address for correspondence: \\ Dr. Amit Thapa \\ Professor and Head of Department, Department of \\ Neurosurgery \\ Kathmandu Medical College Teaching Hospital \\ Sinamangal, Kathmandu, Nepal \\ E-mail: dramitthapa@yahoo.com \\ Contact number: +9779851177995
}

Date of submission: $1^{\text {st }}$ December 2019

Date of acceptance: $19^{\text {th }}$ December 2019

\begin{tabular}{l} 
DOI: https://doi.org:10.3126/njn.v16i3.27333 \\
HOW TO CITE \\
Thapa A. CRASH 3 Trial: "Verum finalem" for Role of \\
Tranexamic Acid in Brain Trauma?. NJNS 2019;16(3):1-5 \\
\hline
\end{tabular}

To access Nepal Journal of Neuroscience Archives, scan $Q R$ code:

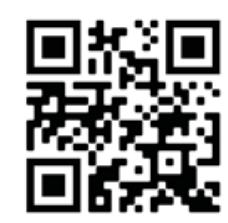

\section{What is CRASH 3 trial?}

Recently CRASH 3 collaborators published the results of their large double blind randomized clinical trial (RCT) done across 175 hospitals regarding the use of tranexamic acid in isolated traumatic brain injuries (TBI) ${ }^{1}$. 12,737 adults with a mean age of 41.7 years (SD 19.0) were randomized to receive either a standardized regimen of tranexamic acid (TXA) (loading dose $1 \mathrm{~g}$ over $10 \mathrm{~min}$ then infusion of $1 \mathrm{~g}$ over $8 \mathrm{~h}$ ) (6406 patients) or an identical placebo (6331 patients).

The outcome studied in CRASH 3 was primarily head injury-related death in hospital within 28 days of injury and did not study radiological change in size of hematoma or side effects of TXA. Secondary outcomes studied were early head injury related death (within $24 \mathrm{~h}$ after injury), all cause and cause specific mortality, disability, vascular occlusive events (myocardial infarction, stroke, deep vein thrombosis, and pulmonary embolism), seizures, complications, neurosurgery, days in the intensive care unit, and adverse events within 28 days of randomization. All analysis was performed on the basis of intention to treat.

In patients who received TXA within 3 hours of TBI, the risk of head injury related mortality was $18.5 \%$ as compared to $19.8 \%$ in the placebo group (855 vs 892 events; risk ratio [RR] 0.94 [95\% CI 0.86-1.02]). However, after excluding patients with Glasgow Coma Scale of 3 with bilateral fixed pupils, TXA group had $12.5 \%$ head injury related mortality compared to $14 \%$ in the placebo group (485 vs 525 events; RR 0.89 [95\% CI $0 \cdot 80-1 \cdot 00]$ ). This reduction in risk of head injury related mortality was significant in patients with mild to moderate TBI (RR 0.78 [95\% CI 0.64-0.95]). In the same group, early administration of TXA was found to more effective $(p=0.005)$. Among patients with reactive pupils, head injury related deaths were found to be lower with the use of tranexamic acid $(0 \cdot 87,[0 \cdot 77-0 \cdot 98])$.

Unfortunately in patients with Severe TBI, early treatment with TXA (even within 3 hours of injury) did not give any statistically significant benefit in reducing head injury related mortality. However, the trial did not find any significant increase in disability among the survivors.

With the use of TXA, CRASH 3 trial did not find any significant increase in risk of vaso-occlusive events (RR $0.98(0.74-1.28)$ or $(1.09$ [95\% CI $0.90-1 \cdot 33])$ as compared to placebo. 


\section{Research-practice gap}

After publication of any such large properly conducted RCT, usually confusion arises regarding application of its results. This is due to the fact that trial results are valid within certain conditions. Due to many other factors, lot of scientific data generated in research do not get immediately adapted in clinical care, sometimes taking almost a decade and hence does not help immediately improve patient care and treatment ${ }^{2,3}$. Research practice gap or evidence-practice gap or knowing-doing gap are synonyms applied to name the substantial gap which exists between the healthcare that patients receive and the practice that is recommended ${ }^{4,5}$.

Hence before adopting any trial results into practice we have to scientifically consider the level of evidence it forms and grade of recommendation which can be made based on available literature. Moreover, for adopting an intervention, we have to understand its generalized applicability and the complications it can generate in unmonitored or off label usage.

\section{Adoptability of a concept}

Every new concept or technology is seen with expectations of benefits which can be predicted to follow a hype cycle (as shown in Figure 1) and consequently the concept or technology gets adopted gradually in phases. Regarding TXA, due to its effective role in stopping bleed it is being adopted by emergency physicians universally however due to skepticism among the neurosurgeons about its usage in isolated neurosurgical scenarios as well as related complications, adoption currently is slow.

\section{What are other evidences?}

Experiences from war and everyday trauma victim care have taught us few simple and obvious lessons: control bleeding, avoid hypotension ${ }^{6}$ and hypoxia, ${ }^{7}$ or the patient will die! Intracranial bleeding by increasing intracranial pressure and brain herniation, increases risk of mortality and morbidity ${ }^{8}$. Hence controlling bleeding seem to be most relevant in early phase of TBI or in condition

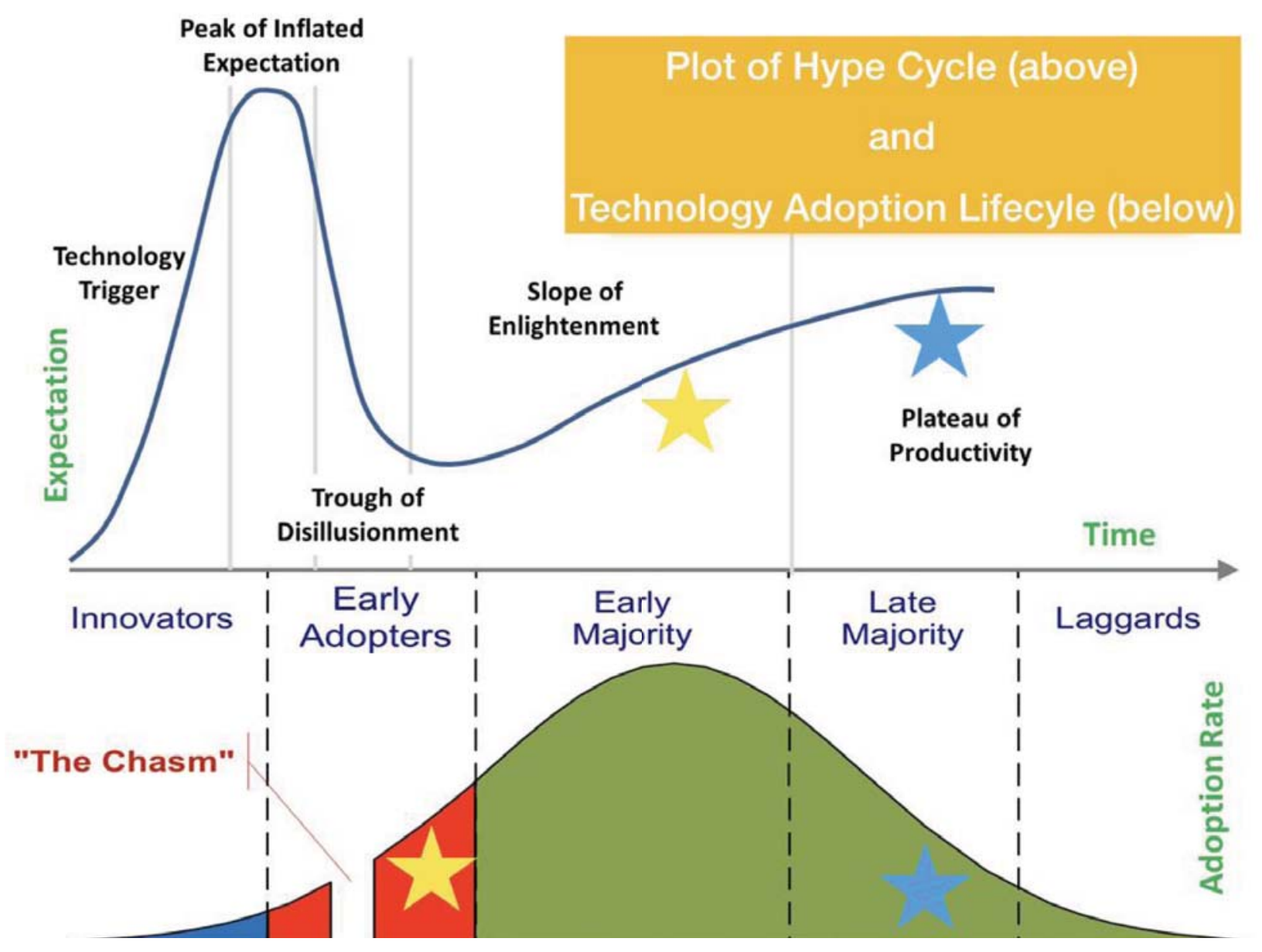

Figure 1. Hype Cycle and adoption of concept. Blue Star represents status of TXA in trauma among emergency physician, however Yellow Star represents status of TXA in isolated TBI among neurosurgeons. 
CRASH 3 trial- verum finalem?

\begin{tabular}{|c|c|c|c|}
\hline \multirow{2}{*}{ Studies, year of publication } & \multicolumn{2}{|c|}{ Head injury related death $\mathrm{n} / \mathrm{N} \%$} & \multirow{2}{*}{$\mathbf{R R}(95 \% \mathbf{C I})$} \\
\hline & Tranexamic acid & Placebo & \\
\hline CRASH $2,2011^{10}$ & $14 / 133(10.5 \%)$ & $24 / 137(17.5 \%)$ & $0.60(0.33-1.11)$ \\
\hline Yutthakasemsunt etal, $2013^{15}$ & $12 / 120(10.0 \%)$ & $18 / 120(15.0 \%)$ & $0.67(0.34-1.32)$ \\
\hline CRASH $3,2019^{1}$ & $485 / 3880(12.5 \%)$ & $525 / 3757(14.0 \%)$ & $0.89(0.80-1.00)$ \\
\hline NCT01990768, $2019^{16}$ & $93 / 603(15.4 \%)$ & $50 / 285(17.5 \%)$ & $0.88(0.64-1.20)$ \\
\hline
\end{tabular}

Table 1: Effect of use of Tranexamic Acid as compared to placebo on head injury related death

with massive bleeding. TXA by inhibiting enzymatic breakdown of fibrin blood clots reduces bleeding.

In 2010, CRASH 2 trial (a study of 20,211 patients with trauma) significantly changed how tranexamic acid was used in trauma victims with significant extra-cranial bleeding as it decreased death due to bleeding by a third when given within 3 hours $^{9,10}$ and the benefit reduces by delaying the treatment ${ }^{11}$. In 2018 Sprigg et al in the TICH-2 trial found decrease in early death with the use of TXA in non-traumatic cerebral hemorrhages, however it did not translate into statistically significant better functional status on day 90 of event ${ }^{12}$. Similarly WOMAN trial of 20,060 patients with peripartum hemorrhage in 2017 did show significantly reduced death due to bleeding with no adverse effects with the use of TXA within 3 hours of birth ${ }^{13}$.

For Isolated TBI, there were small randomized studied suggesting role of TXA in isolated TBI, as described in table $1^{1,9,10,14,15,16}$. The effect may at sometime be subdued due to various dosage model used in trial which may have skewed the results ${ }^{16}$.

\section{What are the strengths of CRASH 3 trial?}

CRASH 3 trial due to its massive sample size and properly conducted blinded randomized format involving investigators, entirely independent of all funding sources, stands out to be genuine and dependable. Blinding the investigators assured non biased outcome assessments. Besides, the trial monitoring unit by directly on-site or remotely auditing patients' record assured reliability of trial data. The reported protocol violation of $0.8 \%$ speaks of high standard of conduct of the study. The statistical analysis of the data was convincing as they analyzed complete case data sets without imputing for missed data. Time to treatment, severity of TBI, systolic blood pressure and age being confounding factors to the effect of TXA, were controlled in multi-variable model.

CRASH 3 trial certainly provides a Level 1 evidence to the use of TXA in isolated brain injury. With the supporting literature of its efficacy (table 1), it can be considered a Grade A recommendation to start TXA in mild to moderate isolated TBI within 3 hours of injury to prevent head injury related mortality and decrease morbidity among the survivors. Its use is not associated with increase in vaso-occlusive events or seizures.

\section{Why did TXA not help severe TBI or avoid late mortalities?}

Severe TBI have many factors besides intracranial bleeding which cumulatively cause death.

The cause of mortality later during the course of treatment of TBI (after 24 hours) like ventilator associated pneumonia or sepsis, may not be directly related to the severity of head injury. These reasons, not related to blood in the brain, are not affected by TXA.

\section{Any shortcoming of CRASH 3 trial?}

The questionable context with the trial was the change in time window for recruitment towards the end of the study, from the original 8 hours to 3 hours. This was based on the pretext that TXA may not be effective if given late after injury based upon a statistical analysis plan before unblinding ${ }^{17}$. Even though trial coordinators admit being blinded to the trial data during this change in protocol, this amendment did put a question mark to the conceptualization of the trial as the sample size was increased to include 13,000 patients from the original 10,000 planned leading to trial extension. For those of us who use TXA during during operative interventions, we do find TXA works wonders if started before the incision! Hence timing is crucial; however this fact was known prior to the start of the trial and should have been incorporated at the start.

The benefit of TXA in mild to moderate TBI was seen only after post hoc analysis by excluding patients with GCS of 3 and fixed dilated pupils. This subset of patients has a poor outcome despite treatment.

Only one dosing regimen of TXA was used in the study irrespective of body weight of the patient, which does not allow evaluation of other dosing regimen as well as creates possibility of low drug level particularly in obese or overweight patients which was not considered in the study.

Since the trial did not regulate the use of other treatment protocols like hypertonic saline or cerebral perfusion 


\section{Thapa et al}

based regimen or hypothermia while managing TBI, such confounding factors which could have affected the study outcome were not taken into consideration.

Regarding the adverse event related to use of TXA, the trial definition of deep vein thrombosis or pulmonary embolism requiring a positive result on imaging (e.g., ultrasound) or at a postmortem examination would have missed clinically minor incidences of vaso-occlusive events in cases.

As the study outcome measures did not look into radiological development of intracranial hematoma with the use of TXA, the mechanism of action of clinical benefits of TXA in isolated TBI is still hypothetical.

\section{What is its implication for Nepal?}

Of the 29 countries 7 centers with neurosurgical facilities from Nepal contributed 255 patients to the trial. As the trial adopted a pragmatic approach regarding the use of TXA and did not influence the associated treatment strategies of the patients, the results do reflect upon the benefit one can get from early use of TXA in our scenario. Since CT was not absolutely necessary (as the patients with Glasgow coma scale of less than 12 were recruited in the study), the benefit of giving TXA as early as possible seems achievable in our context where patients cannot be transported to tertiary care centers on time! The applicability of this study in our scenario is supported by the fact that the effect of tranexamic acid on head injury related death stratified by World Bank income group (high income vs. low income and middle income countries) was not statistically significant $(\mathrm{p}=0.26)$ in the study.

TBI is a major cause of mortality and morbidity in Nepal and with the use of TXA within 3 hours of injury, it seems the head injury related mortality can be reduced without any significant side effect of the TXA and may also reduce the disease burden. Due to resource constraint scenario where all of the patients could not receive immediate neurosurgical services or a CT scan study, it is advisable to use a cheaper drug like TXA as early as possible in patients with GCS of less than 12 .

With over 53,000 patients studied in aforementioned trials for role of TXA in cases of bleeding, one thing is certain TXA does give early survival benefits. Large properly conducted trial like CRASH 3 has provided "verum finalem"on the use of TXA in isolated traumatic brain injury.

\section{References}

1. The CRASH-3 trial collaborators. Effects of tranexamic acid on death, disability, vascularocclusive events and other morbidities in patients with acute traumatic brain injury (CRASH-3): a randomised, placebo-controlled trial. Lancet. 2019;394:1713-23. DOI: 10.1016/ S0140-6736(19)32233-0

2. Brown D, McCormack B. Developing Postoperative Pain Management: Utilising the Promoting Action on Research Implementation in Health Services (PARIHS) Framework. Worldviews Evid Based Nurs, Third Quarter. 2005;2(3):131-41. DOI: 10.1111/j.1741-6787.2005.00024.x

3. Rangachari P, Rissing P, Rethemeyer K. Awareness of evidence-based practices alone does not translate to implementation: insights from implementation research.Qual ManagHealth Care.2013;22(2): 117 25. DOI: 10.1097/QMH.0b013e31828bc21d

4. Pfeffer J, Sutton RI. The Knowing-Doing Gap: how smart companies turn knowledge into action. Boston: Harvard Business School Press; 2000.

5. Real K, Poole MS. Innovation Implementation: Conceptualization And Measurement In Organizational Research. Research in Organizational Change and Development, Volume 15. Bingley: Emerald Group Publishing Limited; 2005.

6. Spaite DW, Hu C, Bobrow BJ, et al. Mortality and prehospital blood pressure in patients with major traumaticbraininjury: implications forthehypotension threshold. JAMA Surg. 2017;152:360-8. DOI: 10.1001/jamasurg.2016.468

7. Spaite DW, Hu C, Bobrow BJ, et al. The effect of combined out-of-hospital hypotension and hypoxia on mortality in major traumatic brain injury. Ann Emerg Med. 2017;69:62-72. DOI: 10.1016/j. annemergmed.2016.08.007

8. Perel P, Roberts I, Bouamra O, Woodford M, Mooney J, Lecky F. Intracranial bleeding in patients with traumatic brain injury: a prognostic study. BMC Emerg Med. 2009;9:15. DOI: 10.1186/1471-227X9-15

9. The CRASH-2 collaborators. Effects of tranexamic acid on death, vascular occlusive events, and blood transfusion in trauma patients with significant haemorrhage (CRASH-2): a randomised, placebo $\neg$ controlled trial. Lancet. 2010;376:23-32. DOI: $10.1016 / \mathrm{S} 0140-6736(10) 60835-5$

10. The CRASH-2 collaborators. The importance of early treatment with tranexamic acid in bleeding trauma patients: an exploratory analysis of the CRASH-2 randomised controlled trial. Lancet. 2011;377:1096101. DOI: 10.1016/S0140-6736(11)60278-X

11. Gayet-Ageron A, Prieto-Merino D, Ker K, Shakur H, Ageron F, Roberts I. Effect of treatment delay on the effectiveness and safety of antifibrinolytics in acute severe haemorrhage: a meta-analysis of individual 


\section{CRASH 3 trial- verum finalem?}

patient-level data from 40138 bleeding patients. Lancet. 2018;391:125-32. DOI: 10.1016/S01406736(17)32455-8

12. Sprigg N, Flaherty K, Appleton JP, Al-Shahi Salman $\mathrm{R}$, Bereczki D, Beridze M, et al. Tranexamic acid to improve functional status in adults with spontaneous intracerebral haemorrhage: the TICH-2 RCT. Health Technol Assess. 2019 Jul;23(35):1-48. DOI: 10.3310/hta23350

13. Woman Trial Collaborators. Effect of early tranexamic acid administration on mortality, hysterectomy, and other morbidities in women with post-partum haemorrhage (WOMAN): an international, randomised, double-blind, placebo-controlled trial. Lancet. 2017;389:2105-16. DOI: 10.1016/S01406736(17)30638-4

14. $\mathrm{CRASH} \neg 2$ (Clinical Randomisation of an Antifibrinolytic in Significant Haemorrhage) intracranial bleeding study: the effect of tranexamic acid in traumatic brain injury - a nested randomised, placebo-controlled trial. Health Technol Assess. 2012;16:1-54. DOI: 10.3310/hta16130

15. Yutthakasemsunt S, Kittiwatanagul W, Piyavechvirat P, Thinkamrop B, Phuenpathom N, Lumbiganon P. Tranexamic acid for patients with traumatic brain injury: a randomized, double-blinded, placebocontrolled trial. BMC Emergency Medicine. 2013;13:20. DOI: 10.1186/1471-227X-13-20

16. May S. Prehospital tranexamic acid use for traumatic brain injury (TXA). Clinical Trials.gov identifier: NCT01990768. [Cited on 15-12-2019]. Available at https://clinicaltrials.gov/ct2/show/NCT01990768

17. Roberts I, Belli A, Brenner A et al. Tranexamic acid for significant traumatic brain injury (the CRASH-3 trial): statistical analysis plan for an international, randomised, double blind, placebo controlled trial. Wellcome Open Res. 2018;3:86. DOI: 10.12688/ wellcomeopenres. 14700.2 